\title{
Low neutrophil-lymphocyte ratio correlates with extended survival in patients with metastatic breast cancer who achieved clinically complete response following multidisciplinary therapy: A retrospective study
}

\author{
HARUKO TAKUWA $^{1}$, WAKAKO TSUJI ${ }^{1}$, YOSHIHIRO YAMAMOTO ${ }^{2}$, \\ MASAYUKI SHINTAKU ${ }^{2}$ and FUMIAKI YOTSUMOTO ${ }^{1}$ \\ Departments of ${ }^{1}$ Breast Surgery and ${ }^{2}$ Pathology, Shiga Medical Center for Adults, Moriyama-shi, Shiga 524-8524, Japan
}

Received November 7, 2017; Accepted February 23, 2018

DOI: $10.3892 / \mathrm{ol} .2018 .8145$

\begin{abstract}
The prognosis of patients with metastatic or recurrent breast cancer (MBC) is improving as novel treatments are developed. The present study compared the clinical characteristics of patients with $\mathrm{MBC}$ with or without a complete clinical response (cCR) and identified the survival-associated factors. This was a retrospective study, which included 171 patients treated for MBC between 2011 and 2017 at the Shiga Medical Center for Adults. Neutrophil to lymphocytes ratios (NLRs) were determined in blood samples. The median follow-up period following diagnosis of MBC was 44 months (range, 0-217 months). A total of 32 patients (18.7\%) achieved a cCR. Compared with the non-cCR group, the cCR group had significantly fewer metastases or recurrences $(\mathrm{P}<0.001)$, significantly fewer visceral metastases $(\mathrm{P}<0.001)$, a significantly lower NLR $(\mathrm{P}<0.001)$ and were diagnosed with primary breast cancer at a significantly earlier stage $(\mathrm{P}=0.003)$. Prognosis was significantly improved in the cCR group compared with the non-cCR group $(\mathrm{P}<0.001)$ and a high NLR $(\geq 19)$ independently predicted worse survival in a multivariate analysis $(\mathrm{P}=0.0218$; hazard ratio, $1.75 ; 95 \%$ confidence interval, 1.09-2.85). In conclusion, the present study
\end{abstract}

Correspondence to: Dr Haruko Takuwa, Department of Breast Surgery, Shiga Medical Center for Adults, 5-4-30 Moriyama, Moriyama-shi, Shiga 524-8524, Japan

E-mail: st24057@yahoo.co.jp

Abbreviations: cCR, clinical complete response; ER, estrogen receptor; HER2, human epithelial growth factor receptor 2; MBC, metastatic breast or recurrent breast cancer; NLR, neutrophil-lymphocyte ratio; OS, overall survival; TIL, tumor-infiltrating lymphocyte

Key words: clinical complete response, metastatic breast cancer, multidisciplinary therapy, neutrophil-lymphocyte ratio, no evidence of disease, tumor-infiltrating lymphocytes determined that achieving a cCR and having a low NLR are important for the long-term survival of patients with MBC.

\section{Introduction}

Approximately 20-30\% of breast cancers ultimately metastasize or recur. The treatments for metastatic or recurrent breast cancer (MBC) are diverse, and treatment innovations have improved the prognosis and life expectancy of MBC patients (1). More than 20 years ago, only 2-3\% of MBC patients achieved a clinical complete response (cCR) and the 10-year survival rate was only about 5\% (2-5), http://ganjoho.jp/reg_stat/). Today, the 10 -year survival rate of $\mathrm{MBC}$ is $15.6 \%$, and the 5-year survival rate is $32.6 \%$, according to the Research Group of the Japanese National Cancer Research Center (6).

Treatment innovations in the past 10 years include the use of small molecule inhibitors and anti-human epidermal growth factor receptor 2 (HER2) antibodies. In recent clinical trials including anti-HER2 therapy, 10-20\% of patients with metastatic breast cancer achieved a cCR (7-9). However, the factors responsible for a cCR are not known, nor is it known when patients discontinue treatment once a cCR is achieved. Owing to potential adverse events, unnecessary treatments should be avoided.

In recent years, many research groups have investigated the value of anticancer immune responses and the hematological components of the systemic inflammatory response specifically for use in predicting outcome. Some studies have evaluated the prognostic and predictive importance of tumor-infiltrating lymphocytes (TILs) in breast cancer $(10,11)$. And some have reported that the combination of the hematological components of the systemic inflammatory response, as the neutrophil-lymphocyte ratio (NLR) have prognostic value in a variety of cancers (12-16).

Therefore, the aim of this study is to analyze the association between cCR and overall survival (OS), and TILs or NLR might be prognositic factor in metastatic breast cancer.

\section{Patients and methods}

Patients. A hundred and seventy-one patients with histologically or clinically confirmed MBC who were consecutively 
treated at the Shiga Medical Center for Adults (Moriyama, Shiga, Japan) between 2011 and 2017 (Table I). Patients had either de novo MBC, a recurrence of a local breast cancer, or distant metastases that appeared after treatment of the primary cancer. Medical records were reviewed in detail. Patients who achieved a cCR were defined as those with no evidence of disease after treatment for MBC (i.e., no evidence of clinical or radiological disease according to the Response Evaluation Criteria in Solid Tumors and as evaluated via computed tomography, magnetic resonance imaging, or positron emission tomography). The frequency and modality of radiographic imaging were at the discretion of the treating physician.

In patients with primary stage IV disease, the abundance of TILs was approximated by examining hematoxylin- and eosin-stained tumor samples under medium power (100x). This examination was limited to patients with stage IV disease because they did not receive prior treatments, which might have affected the TIL score. All samples were reviewed by pathologists. TIL score was defined as the percentage of the tumor and adjacent stroma area infiltrated by lymphocytes; the scores were classified as low $(<10 \%)$, intermediate $(\geq 10 \%$, $50 \%>)$ or high $(\geq 50 \%)(10,11)$. Immunohistochemistry was performed to identify the antigens (CD4 and CD8) in the cell membranes of the TILs. Furthermore, neutrophils are easily affected by factors like infection or therapeutic exposure. In order to minimize the effects of treatment or tumor progression, NLRs were determined in blood samples at diagnosis.

The study design was approved by Ethics Review Board of Shiga Medical Center for Adults according to the Declaration of Helsinki.

Statistics. Qualitative data were examined for differences between the cCR and non-cCR groups; both patient and tumor characteristics were examined, and the chi-square test was used. OS was defined as the interval between the date of diagnosis and the date of the last follow-up or death from any cause. OS was calculated using Kaplan-Meier estimates, and differences in OS were evaluated using the log-rank test. A P-value $<0.05$ was considered significant. A multivariable Cox proportional hazards regression model was used to identify OS-associated factors. To estimate effects of each factor, hazard ratios with $95 \%$ confidence intervals were calculated. Data were analyzed using Stat Mate V for Win \& Mac Hybrid software (ATMS Co., Ltd, Tokyo, Japan).

\section{Results}

The median follow-up time for the 171 patients with $\mathrm{MBC}$ in our study was 44 months (range, 0-271 months). Thirty-two (18.7\%) patients, including 10 patients with HER2 ${ }^{+}$-disease $(5.8 \%)$, had a cCR, with no evidence of disease or a secondary recurrence for 40 months (range, 0-200 months); no patient died during 40 months. All cCR patient terminated treatment after the first or second line of MBC therapy. Most of them had multiple metastatic sites, limited to median 2 organs (range, 1-3 organs). The median time to achieve a cCR was 20 months (range, $0-85$ months). Although patients who had achieved cCR included patients who had undergone metastatic site resection without systemic therapy, usually their main therapy was systemic therapy. Compared with
non-cCR patients, cCR patients had fewer sites of metastases or recurrences $(\mathrm{P}<0.001)$, fewer visceral metastases $(\mathrm{P}<0.001)$, and a lower NLR $(\mathrm{P}<0.001)$ and were diagnosed with primary breast cancer at an earlier stage $(\mathrm{P}=0.003)$.

Among the $120(70.2 \%)$ patients with visceral metastases, 7 patients $(5.8 \%)$ achieved a cCR: 5 patients received systemic therapy without surgery, and 2 patients underwent resection for brain and lung metastases, respectively. In patients with visceral metastases, the NLR at diagnosis was significantly lower in the cCR $(n=7)$ than the non-cCR group $(n=25$, $\mathrm{P}<0.001)$. The characteristics of patients with visceral metastasis are summarized in Table II.

Median OS were longer in cCR group than non-cCR group $(\mathrm{P}<0.001$; Fig. 1). OS was also longer in patients with a low $\operatorname{NLR}(<1.9)$ than in those with a high $\operatorname{NLR}(\geq 1.9)$ at the time of MBC diagnosis (33 vs. 79 months, $\mathrm{P}=0.004$; Fig. 2 ). In the multivariate analysis, a high NLR was associated with worse OS $(\mathrm{P}=0.0218$; hazard ratio, $1.75 ; 95 \%$ confidence interval, 1.09-2.85; Table III). Three patients with a high NLR achieved a cCR, none of three had visceral metastases, and all of them received multidisciplinary therapy consisting of systemic therapy and local resection.

Core needle biopsy samples were obtained from 26 stage IV MBC patients before treatment (Table IV). Two patients had synchronous bilateral breast cancers. Lymphocyte infiltration was scored as high $[\geq 50 \%$; (Fig. $3 \mathrm{~A})$, intermediate $(\geq 10 \%$, $50 \%>$ ), and low ( $<10 \%$; (Fig. 3B)]. Focusing on 4 patients with triple-negative disease, all of them belonged to low TILs and resulted in non-cCR.

\section{Discussion}

MBC accounts for most breast cancer-associated deaths. However, some patients with MBC achieve a cCR and survive for a long time after multidisciplinary treatment. Owing to new agents and therapies, the prognosis for $\mathrm{MBC}$ has been improving (1).

In the present study, patients who achieved a cCR survived for a longer period of time than those who did not (Fig. 1). Compared with patients in the non-cCR group, those in the cCR group were diagnosed with primary breast cancer at an earlier stage and had fewer number of recurrent or metastatic sites, and a lower NLR (Table I). Over half of the patients in cCR group acquired NED status after local resection of lymph node metastases or oligometastases. Thus, volume reduction is an instrumental in achieving a cCR, irrespective of phenotype or Ki-67 status. Most important strategy is appropriate primary disease control. Table II shows that the cCR group tended to have a small number of metastatic sites and a low NLR, even if visceral metastases were present. Seven patients who had a cCR had visceral metastases, 5 of 7 received systemic therapy without surgery. Although the number of patients with visceral metastases who achieved a cCR is small, these patients are expected to increase along with new drugs development. Since trastuzumab was developed in the 1990's, improvement of anti-HER2 therapy has been remarkable. An increase of the patients who achieve a cCR, especially HER2 $2^{+}$patients, is expected in the future.

Our study verified the prognostic value of NLR in MBC, as reported by others (12-16). Additionally, patients with a high 
Table I. Characteristics of patients with metastatic or recurrent breast cancer.

\begin{tabular}{|c|c|c|c|c|}
\hline Variable & All patients $(\mathrm{n}=171)$ & $\mathrm{cCR}(\mathrm{n}=32)$ & non-cCR (n=139) & P-value \\
\hline Follow-up period (months) & & & & 0.135 \\
\hline Median & 44 & 60 & 47 & \\
\hline Range & $0-271$ & $1-247$ & $0-271$ & \\
\hline Age at primary breast cancer (y.o.) & & & & 0.142 \\
\hline Median & 55 & 52 & 55 & \\
\hline Range & $29-89$ & $32-75$ & $29-89$ & \\
\hline Age at metastatic breast cancer (y.o.) & & & & 0.232 \\
\hline Median & 59 & 57 & 59 & \\
\hline Range & $31-92$ & $32-81$ & $31-92$ & \\
\hline Disease stage at primary diagnosis, no. $(\%)$ & & & & 0.003 \\
\hline Stage 0 & $4(2.3)$ & $2(6.3)$ & $2(1.4)$ & \\
\hline Stage I & $24(14.0)$ & $11(34.3)$ & $13(9.4)$ & \\
\hline Stage II & $42(24.6)$ & $5(15.6)$ & $37(26.6)$ & \\
\hline Stage III & $46(27.0)$ & $8(25.0)$ & $38(27.3)$ & \\
\hline Stage IV & $38(22.2)$ & $4(12.5)$ & $34(24.5)$ & \\
\hline Unknown & $17(9.9)$ & $2(6.3)$ & $15(10.8)$ & \\
\hline Histology, no. (\%) & & & & 0.619 \\
\hline Invasive ductal & $148(86.4)$ & $30(93.8)$ & $118(84.9)$ & \\
\hline Invasive lobular & $7(4.1)$ & $0(0.0)$ & $7(5.0)$ & \\
\hline Mixed & $3(1.8)$ & $0(0.0)$ & $3(2.2)$ & \\
\hline Sarcoma & $1(0.6)$ & $0(0.0)$ & $1(0.7)$ & \\
\hline Other & $9(5.3)$ & $2(6.2)$ & $7(5.0)$ & \\
\hline Unknown & $3(1.8)$ & $0(0.0)$ & $3(2.2)$ & \\
\hline Receptor status, no. (\%) & & & & 0.358 \\
\hline ER+/HER2- & $93(54.4)$ & $19(59.4)$ & $74(53.2)$ & \\
\hline $\mathrm{ER}+/ \mathrm{HER} 2+$ & $23(13.5)$ & $4(12.5)$ & $19(13.7)$ & \\
\hline ER-/HER2+ & $20(11.7)$ & $6(18.8)$ & $14(10.1)$ & \\
\hline ER-/HER2- & $28(16.4)$ & $2(6.3)$ & $26(18.7)$ & \\
\hline Unknown & $7(4.0)$ & $1(3.0)$ & $6(4.3)$ & \\
\hline Ki-67 labeling index & & & & 0.885 \\
\hline Median, SD & $20.3 \pm 19.6$ & $20.5 \pm 23.7$ & $20.9 \pm 18.6$ & \\
\hline Range & $1.5-90$ & $1.5-90$ & $2-80$ & \\
\hline Site No. of metastasis/recurrence & & & & $<0.001$ \\
\hline 1 & $52(30.4)$ & $25(78.1)$ & $27(19.4)$ & \\
\hline 2 & $48(28.1)$ & $6(18.8)$ & $42(30.2)$ & \\
\hline$\geq 3$ & $71(41.5)$ & $1(3.1)$ & $70(50.4)$ & \\
\hline No. of visceral metastasis PgR status & & & & $<0.001$ \\
\hline 0 & $51(29.8)$ & $25(78.1)$ & $26(18.7)$ & \\
\hline 1 & $76(44.5)$ & $6(18.8)$ & $70(50.4)$ & \\
\hline 2 & $40(23.4)$ & $1(3.1)$ & $39(28.1)$ & \\
\hline 3 & $4(2.3)$ & $0(0.0)$ & $4(2.8)$ & \\
\hline NLR at diagnosis of metastasis/recurrence & & & & $<0.001$ \\
\hline Median, SD & $2.44 \pm 1.97$ & $1.46 \pm 0.35$ & $2.66 \pm 2.16$ & \\
\hline Range & $0.83-17.50$ & $0.93-2.77$ & $0.83-17.50$ & \\
\hline
\end{tabular}

Bold type indicates a statistically significant difference $(\mathrm{P}<0.005)$. cCR, clinical complete response; ER, estrogen receptor; HER2, human epidermal growth factor receptor 2; NLR, neutrophil-lymphocyte ratio. 
Table II. Characteristics of patients with visceral metastases.

\begin{tabular}{|c|c|c|c|c|}
\hline Variables & All patients $(n=120)$ & $\mathrm{cCR}(\mathrm{n}=7)$ & non-cCR $(n=113)$ & P-value \\
\hline Follow-up period (months) & & & & 0.153 \\
\hline Median & 44 & 98 & 40 & \\
\hline Range & $0-247$ & $13-247$ & $0-171$ & \\
\hline Age at primary breast cancer (y.o.) & & & & 0.222 \\
\hline Median & 55 & 49 & 55 & \\
\hline Range & $29-89$ & $32-61$ & $29-89$ & \\
\hline Age at metastatic breast cancer (y.o.) & & & & 0.077 \\
\hline Median & 59 & 50 & 59 & \\
\hline Range & $31-89$ & $32-61$ & $31-89$ & \\
\hline Disease stage at primary diagnosis, no. (\%) & & & & 0.942 \\
\hline Stage 0 & $2(1.7)$ & $0(0.0)$ & $2(1.8)$ & \\
\hline Stage I & $10(8.3)$ & $1(14.2)$ & $9(8.0)$ & \\
\hline Stage II & $33(27.5)$ & $2(28.6)$ & $31(27.4)$ & \\
\hline Stage III & $33(27.5)$ & $2(28.6)$ & $31(27.4)$ & \\
\hline Stage IV & $30(25.0)$ & $2(28.6)$ & $28(24.8)$ & \\
\hline Unknown & $12(10.0)$ & $0(0.0)$ & $12(10.6)$ & \\
\hline Histology, no. (\%) & & & & 0.964 \\
\hline Invasive ductal & $106(88.4)$ & $7(100.0)$ & $99(87.6)$ & \\
\hline Invasive lobular & $4(3.3)$ & $0(0.0)$ & $4(3.5)$ & \\
\hline Mixed & $1(0.8)$ & $0(0.0)$ & $1(0.9)$ & \\
\hline Sarcoma & $1(0.8)$ & $0(0.0)$ & $1(0.9)$ & \\
\hline Other & $6(5.0)$ & $0(6.2)$ & $6(5.3)$ & \\
\hline Unknown & $2(1.7)$ & $0(0.0)$ & $2(1.8)$ & \\
\hline Receptor status, no. (\%) & & & & 0.983 \\
\hline ER+/HER2- & $65(54.2)$ & $4(57.1)$ & $61(54.0)$ & \\
\hline ER+/HER2+ & $15(12.5)$ & $1(14.3)$ & $14(12.4)$ & \\
\hline ER-/HER2+ & $14(11.7)$ & $1(14.3)$ & $13(11.5)$ & \\
\hline ER-/HER2- & $22(18.3)$ & $1(14.3)$ & $21(18.6)$ & \\
\hline Unknown & $4(3.3)$ & $0(0.0)$ & $4(3.5)$ & \\
\hline Ki-67 labeling index & & & & 0.352 \\
\hline Median \pm SD & $22.3 \pm 19.6$ & $13.3 \pm 20.6$ & $23.2 \pm 18.9$ & \\
\hline Range & $1.5-80$ & $1.5-50$ & $2-80$ & \\
\hline Site no. of metastasis/recurrence & & & & 0.006 \\
\hline 1 & $17(14.2)$ & $3(42.9)$ & $14(12.4)$ & \\
\hline 2 & $36(30.0)$ & $4(57.1)$ & $32(28.3)$ & \\
\hline$\geq 3$ & $67(55.8)$ & $0(0.0)$ & $67(59.3)$ & \\
\hline NLR at diagnosis of metastasis/recurrence & & & & $<0.001$ \\
\hline Median \pm SD & $2.84 \pm 2.31$ & $1.29 \pm 0.10$ & $2.92 \pm 2.34$ & \\
\hline Range & $0.83-17.50$ & $1.21-1.40$ & $0.83-17.50$ & \\
\hline
\end{tabular}

Bold type indicates a statistically significant difference $(\mathrm{P}<0.01)$. cCR, clinical complete response; ER, estrogen receptor; HER2, human epidermal growth factor receptor 2; NLR, neutrophil-lymphocyte ratio.

NLR achieved a cCR by multidisciplinary therapy combined with, local resection and systemic therapy. Neutrophils play an important role in the metastatic microenvironment (17-19). It is generally believed that neutrophils dynamically regulate cancer progression and metastasis. Resection of metastatic sites where the immune system does not target cancer cells is a reasonable strategy.
To assess the relationship between the tumor microenvironment and therapeutic effects, we focused on TILs because a high serous NLR might reflect local lymphocyte invasion. We examined lymphocyte infiltration in patients with stage IV disease (Table IV); because they were treatment-naïve, and their TIL scores were treatment-unrelated. Twenty-eight specimens from 26 patients were available for review. Assessment 


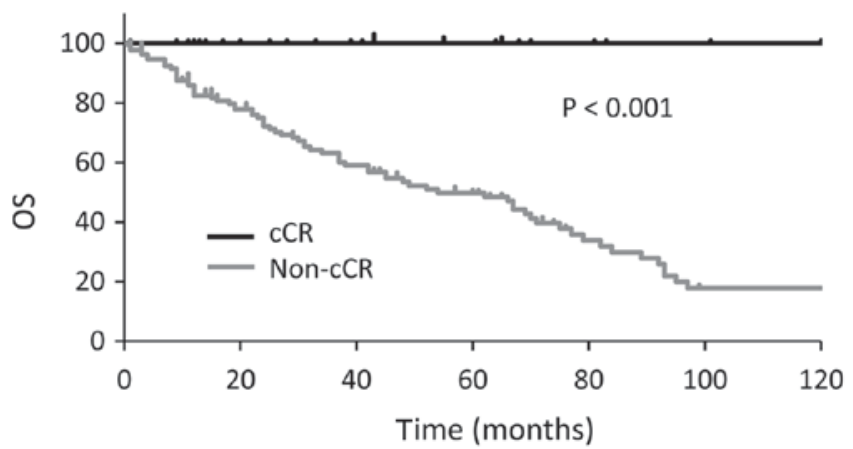

Figure 1. Kaplan-Meier plot comparing OS times between the cCR group and the non-cCR group. OS, overall survival; cCR, clinical complete response.

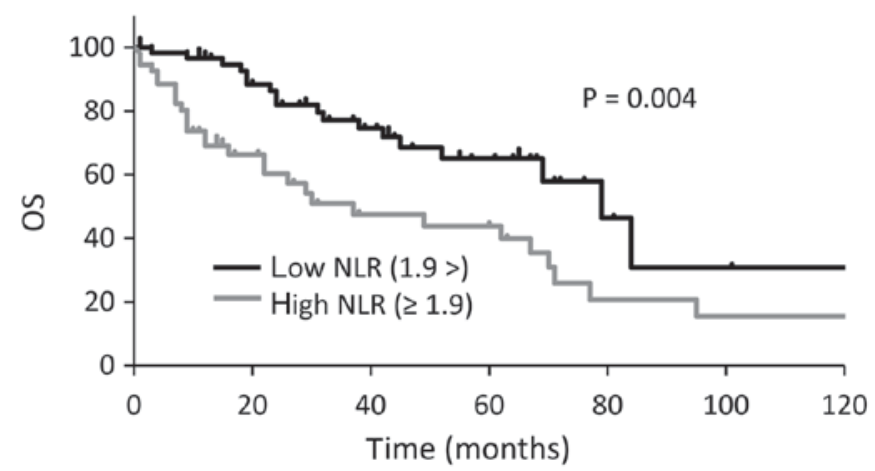

Figure 2. Kaplan-Meier plot comparing OS times between patients with a low NLR (<1.9) and patients with a high NLR ( $\geq 1.9)$. OS, overall survival; NLR, neutrophil-lymphocyte ratio.

of the 4 patients ( 3 low TILs; 1 high TILs) who achieved a cCR [estrogen receptor $(\text { ER })^{+} / \mathrm{HER}^{-}, 2$ patients; ER $/$ HER2 ${ }^{+}$, 2 patients] showed that the TIL score had no prognostic value in MBC. According to previous reports, the TIL score is a prognostic marker in HER2 ${ }^{+}$breast cancers (20), as well as triple-negative breast cancers (TNBC) in the both neoadjuvant and adjuvant settings $(11,21,22)$. Because all TNBC patients in this study showed low TILs, the relationship between prognosis of TNBC patients and high TILs could not be evaluated.

We did not evaluate the biopsy samples from all metastatic sites. This would be of interest because metastatic cancer cells have different characteristics from primary cancer cells (23). Additionally, TILs review was performed in core needle biopsy samples histologically. Strictly, core needle biopsy was not standard approarch for TILs evaluation (10).

In our study, 43 of the 137 patients with primary stage I-III breast cancer experienced recurrence during adjuvant therapy; the phenotypes of tumors were ER $/$ HER2- (29 patients), $\mathrm{ER}^{+} / \mathrm{HER}^{+}$(9 patients), ER $/ \mathrm{HER}^{+}{ }^{+}$(1 patient), and ER $/ \mathrm{HER} 2$ (4 patients). In these patients, recurrence is thought to be mainly from tumor-related factors (e.g., resistance to systemic therapy) rather than host-related factors. Because recent whole-exosome and transcriptome analysis revealed that one of the most important mechanism in acquired drug resistance in breast cancer therapy is mutation in cancer cells, not in host normal cells $(24,25)$. Host-related factors such as individual adherence to therapy, ability of drug metabolism, activity of drug degrading enzyme are also important. However, appropriate
Table III. Multivariate analysis of factors associated with overall survival.

\begin{tabular}{lccc}
\hline Variable & HR & $95 \%$ CI & P-value \\
\hline Non-cCR & 2.27 & $0.87-5.94$ & 0.0955 \\
Primary stage IV & 1.14 & $0.64-2.03$ & 0.6495 \\
Metastatic sites no. $\geq 3$ & 1.79 & $0.95-3.36$ & 0.0714 \\
Visceral sites no. $\geq 2$ & 1.07 & $0.62-1.86$ & 0.7968 \\
NLR $\geq 1.90$ & 1.75 & $1.09-2.85$ & $\mathbf{0 . 0 2 1 8}$ \\
\hline
\end{tabular}

Bold type indicates a statistically significant difference $(\mathrm{P}<0.05)$. $\mathrm{HR}$, hazard ratio; CI, confidence interval; cCR, clinical complete response; NLR, neutrophil-lymphocyte ratio.
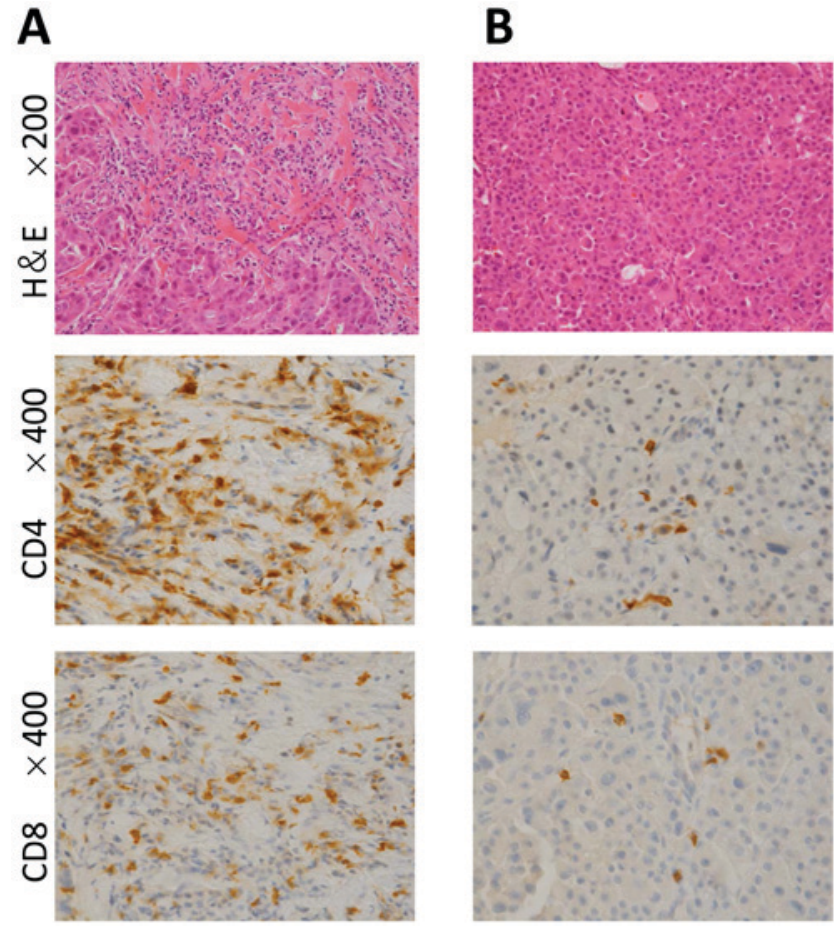

Figure 3. Representative photomicrographs of TILs in two patients with untreated HER2-enriched type stage IV metastatic breast cancer. Each patient achieved a cCR. (A) one had a high TIL score and the other had a (B) low TIL score. Upper images, hematoxylin and eosin stained tissue in a field viewed at medium power (magnification, x200). Middle images, CD4 immunostaining (magnification, $\mathrm{x} 400$ ). Lower images, CD8 immunostaining (magnification, $\mathrm{x} 400$ ). TIL, tumor infiltrating lymphocyte; HER2, human epidermal growth factor receptor 2.

adjuvant systemic therapy is especially needed regarding the high mutation activity of tumor related to drug-resistance.

In conclusion our study showed cCR and low NLRs associate with extended survival times in patients with MBC.

\section{Acknowledgements}

Not applicable.

\section{Funding}

No funding was received. 
Table IV. Clinicopathological implications of TILs for patients with primary stage IV disease.

\begin{tabular}{|c|c|c|c|c|c|c|c|c|}
\hline No. & Age & ER/HER2 & $\mathrm{Ki}-67(\%)$ & Metastatic site & TILs & Outcome & $\begin{array}{c}\mathrm{f} / \mathrm{u} \\
\text { (months) }\end{array}$ & $\begin{array}{c}\text { Other } \\
\text { information }\end{array}$ \\
\hline 1 & 66 & $+/-$ & 20 & Bone, lung, LN & Low & PD & 16 & \\
\hline \multirow[t]{2}{*}{2} & 67 & $+/-($ rt.) & 2 & Lung & Low & $\mathrm{SD}$ & 19 & Bilateral \\
\hline & & +/- (lt.) & 2 & Lung & Low & $\mathrm{SD}$ & 19 & \\
\hline 3 & 65 & $+/-$ & 20 & Lung & Low & SD & 50 & \\
\hline 4 & 85 & $+/-$ & 50 & Lung & Low & PR & 11 & \\
\hline 5 & 59 & $+/-$ & 7.5 & Bone, lung, LN & Intermediate & SD & 15 & \\
\hline 6 & 68 & $+/-$ & 20 & Bone & Low & $\mathrm{cCR}$ & 29 & \\
\hline 7 & 59 & $+/-$ & 5 & Bone, liver, LN & Low & Deceased & 10 & ILC \\
\hline 8 & 89 & $+/-$ & 10 & Bone, lung & Low & Deceased & 10 & ILC \\
\hline 9 & 80 & $+/-$ & 10 & Bone & Low & $\mathrm{PD}$ & 57 & \\
\hline 10 & 56 & $+/-$ & 4 & $\begin{array}{l}\text { Contralateral breast, bone, } \\
\text { pleura, LN, peritoneum }\end{array}$ & Intermediate & Deceased & 7 & ILC \\
\hline 11 & 58 & $+/-$ & l & Bone, pleura, lung & Low & Deceased & 43 & IMPC \\
\hline 12 & 61 & $+/-$ & 40 & Bone, liver & Low & Deceased & 4 & \\
\hline 13 & 61 & $+/-$ & 1.5 & Lung & Low & $\mathrm{cCR}$ & 69 & \\
\hline 14 & 66 & $+/-$ & / & Bone, lung, liver, LN & Low & Deceased & 66 & \\
\hline 15 & 54 & $+/-$ & l & $\begin{array}{l}\text { Bone, pleura, } \\
\text { pericardiac membrane }\end{array}$ & Intermediate & Deceased & 62 & \\
\hline \multirow[t]{2}{*}{16} & 44 & $+/-(\mathrm{rt})$. & 2 & Bone & Intermediate & $\mathrm{PD}$ & 14 & Bilateral \\
\hline & 44 & +/+ (lt.) & 3 & Bone & Intermediate & PD & 14 & \\
\hline 17 & 64 & $+/+$ & 10 & Bone, liver & Low & PR & 3 & \\
\hline 18 & 36 & $+/+$ & 30 & Bone, lung & Low & PR & 19 & \\
\hline 19 & 62 & $-/+$ & 50 & Lung, liver & Intermediate & SD & 48 & \\
\hline 20 & 56 & $-/+$ & 90 & Contralateral LN & Low & $\mathrm{cCR}$ & 14 & Fig. 3B \\
\hline 21 & 32 & $-/+$ & 50 & Lung, LN & High & $\mathrm{cCR}$ & 38 & Fig. 3A \\
\hline 22 & 57 & $-/+$ & 40 & $\begin{array}{l}\text { Bone, pleura, LN, } \\
\text { contralateral } \\
\text { breast, local }\end{array}$ & Intermediate & $\mathrm{PD}$ & 37 & \\
\hline 23 & 75 & $-1-$ & 5 & Bone, lung, liver, muscle & Low & Deceased & 4 & \\
\hline 24 & 87 & $-/-$ & 7.5 & Bone & Low & $\mathrm{SD}$ & 2 & \\
\hline 25 & 60 & $-/-$ & l & Pleura, local & Low & Deceased & 67 & \\
\hline 26 & 85 & $-/-$ & l & Lung, peritoneum, LN & Low & $\mathrm{SD}$ & 1 & \\
\hline
\end{tabular}

TILs, tumor infiltrating lymphocytes; ER, estrogen receptor; HER2, human epidermal growth factor receptor 2; rt., right; lt., left; LN, lymph nodes; cCR, clinical complete response; PR, partial response; SD, stable disease; PD, progressive disease; BC, breast cancer; f/u, follow up; ILC, invasive lobular carcinoma; IMPC, invasive micropapillary carcinoma.

\section{Availability of data and materials}

The datasets used and/or analyzed during the current study are available from the corresponding author on reasonable request.

\section{Authors' contributions}

HT, WT and FY designed the study. MS and YY contributed to the evaluation of TIL scoring and the immunohistochemistry analysis of CD4 and CD8. HT performed survival analysis and multivariate analysis of the other data.

\section{Ethics approval and consent to participate}

The present study was approved by the Ethics Review board of the Shiga Medical Center for Adults.

\section{Consent for publication}

Not applicable.

\section{Competing interests}

The authors declare that they have no competing interests. 


\section{References}

1. Tsuji W, Teramukai S, Ueno M, Toi M and Inamoto T: Prognostic factors for survival after first recurrence in breast cancer: A retrospective analysis of 252 recurrent cases at a single institution. Breast Cancer 21: 86-95, 2014.

2. Giordano SH, Buzdar AU, Smith TL, Kau SW, Yang Y and Hortobagyi GN: Is breast cancer survival improving? Cancer 100 44-52, 2004.

3. Gennari A, Conte P, Rosso R, Orlandini C and Bruzzi P: Survival of metastatic breast carcinoma patients over a 20 -year period: A retrospective analysis based on individual patient data from six consecutive studies. Cancer 104: 1742-1750, 2005.

4. Greenberg PA, Hortobagyi GN, Smith TL, Ziegler LD, Frye DK and Buzdar AU: Long-term follow-up of patients with complete remission following combination chemotherapy for metastatic breast cancer. J Clin Oncol 14: 2197-2205, 1996.

5. Falkson G, Gelman RS, Leone L and Falkson CI: Survival of premenopausal women with metastatic breast cancer. Long-term follow-up of eastern cooperative group and cancer and leukemia group B studies. Cancer 66: 1621-1629, 1990.

6. Rahman ZU, Frye DK, Smith TL, Asmar L, Theriault RL, Buzdar AU and Hortobagyi GN: Results and long term follow-up for 1581 patients with metastatic breast carcinoma treated with standard dose doxorubicin-containing chemotherapy: A reference. Cancer 85: 104-111, 1999.

7. Burstein HJ, Keshaviah A, Baron AD, Hart RD, Lambert-Falls R, Marcom PK, Gelman R and Winer EP: Trastuzumab plusvinorelbine or taxane chemotherapy for HER2-overexpressing metastatic breast cancer: The trastuzumab and vinorelbine or taxane study. Cancer 110: 965-972, 2007.

8. Baselga J, Cortés J, Kim SB, Im SA, Hegg R, Im YH, Roman L, Pedrini JL, Pienkowski T, Knott A, et al: Pertuzumab plus trastumab plus docetaxel for metastatic breast cancer. N Engl J Med 366: 109-119, 2012.

9. Verma S, Miles D, Gianni L, Krop IE, Welslau M, Baselga M, Pegram M, Oh DY, Diéras V, Guardino E, et al: Trastuzumab emtansine for HER2-positive advanced breast cancer. N Engl J Med 367: 1783-1791, 2012.

10. Salgado R, Denkert C,Demaria S, Sirtaine N,Klauschen F, PrineriG, Wienert S, Van den Eynden G, Baehner FL, Penault-Llorca F, et al: The evaluation of tumor-infiltrating lymphocytes (TILs) in breast cancer: Recommendations by an International tils working group 2014. Ann Oncol 26: 259-271, 2015.

11. Hida AI and Ohi Y: Evaluation of tumor-infiltrating lymphocytes in breast cancer; proposal of a simpler method. Ann Oncol 26: 2351,2015

12. Jia W, Wu J, Jia H, Yang Y, Zhang X, Chen K and Su F: The peripheral blood neutrophl-to-lymphocyte ratio is superior to the lymphocyte-to-monocyte ratio for predicting the long-term survival of triple-negative breast cancer patients. PLoS One 10: $\mathrm{e} 0143061,2015$.
13. Orditura M, Galizia G, Diana A, Saccone C, Cobellis L, Ventriglia J, Iovino F, Romano C, Morgillo F, Mosca L, et al: Neutrophil to lymphocyte ratio (NLR) for prediction of distant metastasis-free survival (DMFS) in early breast cancer: A propensity score-matched analysis. ESMO Open 1: e000038, 2016.

14. Iwase T, Sangai T, Sakakibra M, Sakakibra J, Ishigami E, Hayama S, Nakagawa A, Masuda T, Tabe S and Nagashima T: An increased neutrophil-to-lymphocyte ratio predicts poorer survival following recurrence for patients with breast cancer. Mol Clin Oncol 6: 266-270, 2017.

15. Ethier JL, Desautels D, Templeton A, Shah PS and Amir E: Prognostic role of neutrophil-to-lymphocyte ratio in breast cancer. A systematic review and meta-analysis. Breast Cancer Res 19: 2, 2017.

16. Guthrie GJ, Charles KA, Roxburgh CS, Horgan PG, McMillan DC and Clarke SJ: The systemic inflammation-based neutrophil-lymphocyte ratio: Experience in patients with cancer. Crit Rev Oncol Hematol 88: 218-230, 2013.

17. Acharyya S and Massague J: Arresting supporters: Targeting neutrophils in metastasis. Cell Res 26: 273-274, 2016.

18. Bird L: Tumour immunology: Neutrophils help tumours spread. Nat Rev Immunol 16: 74-75, 2016.

19. Wculek SK and Malanchi I: Neutrophils support lung colonization of metastasis-initiating breast cancer cells. Nature 528: 413-417, 2015.

20. Dieci MV, Mathieu MC, Guarneri V, Conte P, Delaloge S, Andre F and Goubar A: Prognostic and predictive value of tumor-infiltrating lymphocytes in two phase III randomized adjuvant breast cancer trials. Ann Oncol 26: 1698-1704, 2015.

21. Castaneda CA, Mittendorf E, Casavilca S, Wu Y, Castillo M, Arboleda P, Nunez T, Guerra H, Barrionuevo C, Dolores-Cerna $\mathrm{K}$, et al: Tumor infiltrating lymphocytes in triple negative breast cancer receiving neoadjuvant chemotherapy. World J Clin Oncol 7: 387-394, 2016.

22. Hida AI, Sagara Y, Yotsumoto D, Kaemitsu S, Kawano J, Baba S, Rai Y, Oshiro Y, Aogi K, Sagara Y and Ohi Y: Prognostic and predictive impacts of tumor-infiltrating lymphocytes differ between Triple-negative and HER2-positive breast cancers treated with standard systemic therapies. Breast Cancer Res Treat 158: 1-9, 2016.

23. Lambert AW, Pattabiraman DR and Weinberg RA: Emerging biological principles of metastasis. Cell 168: 670-691, 2017.

24. Robinson DR, Wu YM, Vats P, Su F, Lonigro RJ, Cao X, Kalyana-Sundaram S, Wang R, Ning Y, Hodges L, et al: Activating ESR1 mutations in hormone-resistant metastatic breast cancer. Nat Genet 45: 1446-1451, 2013.

25. Zardavas D, Phillips WA and Loi S: PIK3CA mutations in breast cancer: Reconciling findings from preclinical and clinical data. Breast Cancer Res 16: 201, 2014. 